\title{
A Peculiar Subclass of Type Ia Supernovae a.k.a. Type Iax
}

\author{
Mridweeka Singh $^{1,4 *}{ }^{,}$Kuntal Misra $^{1}$, Devendra Kumar Sahu ${ }^{2}$, Raya Dastidar ${ }^{1}$, \\ Anjasha Gangopadhyay ${ }^{1,4}$, Subhash Bose ${ }^{3}$, Shubham Srivastav ${ }^{2}$, \\ Gadiyara Chakrapani Anupama ${ }^{2}$, Nand Kumar Chakradhari ${ }^{4}$, Brajesh Kumar ${ }^{1}$, \\ Brijesh Kumar $^{1}$, Shashi Bhushan Pandey ${ }^{1}$
}

\author{
${ }^{1}$ Aryabhatta Research Institute of observational sciencES, Nainital, 263001, India \\ ${ }^{2}$ Indian Institute of Astrophysics, Koramangala, Bangalore, 560 034, India \\ ${ }^{3}$ Kavli Institute for Astronomy and Astrophysics, Peking University, 5 Yiheyuan Road, \\ Haidian District, Beijing 100871, P.R. China \\ ${ }^{4}$ Pt. Ravi Shankar Shukla University, Raipur, 492 010, India
}

\begin{abstract}
We present optical photometric (upto $\sim 410$ days since $B_{\max }$ ) and spectroscopic (upto $\sim 235$ days since $B_{\max }$ ) observations of a type Iax supernova SN 2014dt located in M61. The broad band light curves follow a linear decline upto $\sim 100$ days after which a significant flattening is seen in the late-time (beyond 150 days) light curves of SN 2014dt. SN 2014dt best matches the light curve evolution of SN 2005hk and reaches a peak magnitude of $\mathrm{M}_{B} \sim-18.12 \pm 0.04$ with $\Delta m_{15} \sim 1.35 \pm 0.06$ mag. The earliest spectrum at $\sim 23$ days is dominated by FeII and CoII lines with the absence of the Si II $6150 \AA$ line. Using the peak bolometric luminosity we estimate a ${ }^{56} \mathrm{Ni}$ mass of $0.14 \mathrm{M}_{\odot}$ in the case of SN $2005 \mathrm{hk}$ and the striking similarity between SN 2014dt and SN 2005hk implies that a comparable amount of ${ }^{56} \mathrm{Ni}$ would have been synthesized in the explosion of SN 2014dt. There are several explosion scenarios proposed for these peculiar events. Being one of the brightest and closest SN, SN 2014dt is an ideal candidate for long term monitoring. Late phase observations are very essential to understand the progenitor system and the actual explosion scenario for these events.
\end{abstract}

\section{Introduction}

Type Ia SNe which are hydrogen deficient are the result of the complete disruption of a carbon oxygen white dwarf accreting matter from its companion and are commonly known as thermonuclear SNe. Type Ia SNe have secondary peaks in their I band light curves 30-35 days after maximum in B band. Type Ia SNe exhibit a wide range of similarities and can be parameterized with a few parameters. These parameters are the decline rate $\Delta \mathrm{m}_{15}$ which is the difference in magnitude during 15 days since maximum in B band and $\mathrm{R}$ (Si II) which is the ratio of the depth of two absorption features usually attributed to the Si II 5972 and Si II 6355 lines (Nugent et al. 1995).

Generally it is believed that type Ia SNe originate from the complete eruption of a carbon oxygen white dwarf. But there is a subclass of type Ia SNe which supports a different mechanism of explosion because of

*mridweeka@aries.res.in, yashasvi04@gmail.com 
their peculiar features. Members of this subclass are low luminous and less energetic as compared to normal type Ia SNe. Foley et al.(2013) found that the rate of occurrence of type Iax SNe is $31_{-13}^{+19}$ of every normal 100 type Ia SNe. Li et al. (2011) quote that there are about 5\% SN 2002cx-like objects. Type Iax SNe possess striking differences as compared to normal type Ia as they are devoid of any secondary peaks in their NIR light curves (Li et al. 2003). They exhibit a wide range of around five magnitudes in peak absolute brightness $\left(\mathrm{M}_{B}=\right.$ -14 to $-18 \mathrm{mag})$, their expansion velocities are $\left(\sim 4000\right.$ to $\left.\sim 9000 \mathrm{~km} \mathrm{~s}^{-1}\right)$ half of that of type Ia SNe $(\sim 10,000$ to $\sim 15,000 \mathrm{~km} \mathrm{~s}^{-1}$ ). They are sub-luminous events but their early time spectra are similar to SN 1991T-like over-luminous events. Foley et al.(2013) also showed that type Iax SNe exhibit a correlation between their light curve shape and luminosity but with a large scatter. A weak correlation was also seen between the peak luminosity and the ejecta velocity (Foley et al. 2013). Also the nebular spectra of type Ia SNe are associated with forbidden emission lines of the iron group elements whereas type Iax have a bunch of permitted lines of these iron group elements along with intermediate-mass elements ( $\mathrm{S}, \mathrm{Ca}$, etc.). It is also seen that the late-time spectra of type Iax SNe have calcium interior to iron (Foley et al. 2013) which is opposite to what we see in type Ia SNe. After the discovery of SN 2002cx (Li et al. 2003), a few more SNe have been added in the class of type Iax SNe namely SN 2005hk (Sahu et al. 2008; Phillips et al. 2007), SN 2008ha (Foley et al. 2009), 2010ae (Stritzinger et al. 2014), SN 2011 ay (Szalai et al. 2015), SN 2012Z (Stritzinger et al. 2015), (Yamanaka et al. 2015), SN 2013en (Liu et al. 2015), SN 2015H (Magee et al. 2016) and SN 2014dt (Foley et al. 2015; Fox et al. 2016; Singh et al. 2016 (MNRAS, under revision)). A detailed study of the individual SNe reveals several peculiar features.

\section{OBSERVATIONS AND DATA REDUCTION}

\subsection{Photometry}

A rigorous follow up of SN 2014dt was done with the $104 \mathrm{~cm}$ Sampurnanand Telescope (ST), $130 \mathrm{~cm}$ Devasthal Fast Optical Telescope (DFOT) situated in ARIES, Nainital (India) and $201 \mathrm{~cm}$ Himalayan Chandra Telescope (HCT) situated in Indian Astronomical Observatory (IAO) Hanle (India). The photometric observations of SN 2014dt started 1 day after discovery and lasted upto 400 days. Along with the SN field, bias frames for removing zero integration noise and flat frames for removing non uniformity of pixel to pixel response were also taken. The pre-processing of the data such as bias subtraction, flat fielding were done using the packages available in IRAF ${ }^{1}$. High energetic particles such as cosmic rays were removed by using the task L.A. Cosmic (van Dokkum 2001). To improve the $\mathrm{S} / \mathrm{N}$ ratio wherever multiple frames were available on a single night, images were co-added. Since the SN is situated away from the galaxy centre, near one of the spiral arms of the galaxy, we performed PSF photometry using packages in DAOPHOT II (Stetson 1987) to estimate the SN magnitudes. We took the first aperture equal to the mean FWHM of the frame and inner sky annulus was taken to be four times the FWHM. An eight pixel wide sky annulus was selected with respect to the inner sky annulus. To convert the instrumental magnitudes of SN to standard magnitudes, we used the night to night zero points estimated using the secondary field stars.

\subsection{Spectroscopy}

Long slit low resolution spectroscopic data at 19 epochs were obtained with HCT, equipped with the Hanle Faint Object Spectrograph and Camera (HFOSC). A pair of grisms Gr7 (3800 $\AA-7800 \AA$ ) with a resolution 1330 and Gr8 (5800 $\AA-9200 \AA$ ) with a resolution 2190 is used with HFOSC for spectroscopic observations. A combination of these grisms was taken because most of the metal lines which are seen in a SN spectrum typically fall in these wavelength ranges. The slit width was chosen so as to avoid contamination due to the host. Exposure time was varied between 600 to 1800 seconds in order to obtain good S/N spectra. For

\footnotetext{
${ }^{1}$ IRAF stands for Image Reduction and Analysis Facility distributed by the National Optical Astronomy Observatories which is operated by the Association of Universities for research in Astronomy, Inc., under cooperative agreement with the National Science Foundation.
} 
wavelength calibration arc lamps ( $\mathrm{FeAr}$ and $\mathrm{FeNe}$ ) were observed just after $\mathrm{SN}$ exposure and for flux calibration spectrophotometric standard stars (Feige 34, Feige 110 and HZ 44) were observed each night along with the SN. Necessary pre-processing was done with standard tasks in IRAF whereas for spectral reduction, tasks such as APALL, IDENTIFY, DISPCOR, STANDARD, SENSFUNCTION and CALIBRATE were used. To cross check the wavelength calibration, OI emission lines at 5577, 6300 and $6364 \AA$ were used and in some cases a wavelength shift within 0.2 to $4 \AA$ was found. This shift was applied to correct the spectrum using SPECSHIFT. The blue and red region spectra in Gr7 and Gr8 are combined by applying a scaling factor to get the final spectrum on a relative flux scale. The relative flux spectra are brought to an absolute flux scale by estimating the scale factors from BVRI magnitudes. We have not removed the telluric lines from the spectra. Spectra were corrected for redshift by using DOPCOR.

\section{Temporal evolution and prime spectroscopic features of $\mathrm{SN}$ 2014dt}

Fig. 1 shows the light curve evolution of SN 2014dt in BVRI bands upto $\sim 400$ days after discovery. No secondary peak is seen in I band between 25 - 35 days after $\mathrm{B}_{\max }$ unlike type Ia SNe. We lack observations between 100 days to 160 days after discovery due to unfavorable weather. The photometric monitoring was continued upto 220 days since discovery, after that the object went behind the Sun. Continued follow up observations reveal that the SN was still visible after a year (400 days). SN 2014dt shows some striking similarities as well as differences both photometrically and spectroscopically when compared with the same sub-class of objects. Broad band light curves of SN 2014dt (Fig. 1) exhibit some scattering along with flattening at later phases (after $\sim 150$ days). Typically upto 300 days the light curves of type Ia SNe are governed by the trapping of gamma rays. After that the gamma ray regime transitions to the regime dominated by full positron trapping. Gamma ray trapping may be one of the possibilities for the flattening seen in the light curves of SN 2014dt. Late phase ( $>60$ days) decay rates for SN 2014dt are less than the standard $\mathrm{Co} \rightarrow \mathrm{Fe}$ decay rate which is 0.0098 mag day $^{-1}$. Only the $\mathrm{B}$ band decay rate during late phase is higher than the standard $\mathrm{Co} \rightarrow \mathrm{Fe}$ decay rate. The overall late phase evolution of SN 2014dt indicates towards a flattening which implements gamma ray trapping.

Fig. 2 represents the evolution of the bolometric luminosity of SN 2014dt and its comparison with several well studied type Iax SNe. From that figure it is clear that the luminosity of SN 2014dt is similar to that of SN 2005hk hence we can say that the explosion parameters of SN 2014dt will be comparable to those of SN 2005hk. For SN 2005hk, we estimate ${ }^{56} \mathrm{Ni}$ between 0.14-0.19 $\mathrm{M}_{\odot}$ using Arnett's method (Arnett 1982). Subsequently the ejecta mass and kinetic energy of the explosion in SN 2005hk are $0.98 \mathrm{M}_{\odot}$ and $0.41 \times 10^{51}$ erg. We conclude that the amount of synthesized ${ }^{56} \mathrm{Ni}$ in the explosion of SN $2014 \mathrm{dt}$ would be at least 0.14 $\mathrm{M}_{\odot}$.

Like SN 2002cx, SN 2014dt also has an early phase spectrum similar to SN 1991T but not over luminous. The Fe II and Ca II lines evolution is very prominent in the case of SN 2014dt which implies that the ionization state of these elements is nearly constant and that the photosphere attains a relatively cooler temperature indicating the SN enters into the nebular phase fairly early; the absence of strong Fe III lines strengthens this statement. The last epoch spectrum at 235 days has a poor signal to noise ratio. Last epoch spectrum is dominated by strong emission peaks suggesting a late nebular phase of SN with a weak blue continuum. Spectrum at 23 days after $B_{\max }$ of SN 2014dt is presented in Fig. 3. The velocity evolution feature also confirms the low energy budget of SN 2014dt, a typical feature of peculiar type Ia SNe. Velocity of absorption minima for a number of lines vary between $\sim 1500 \mathrm{~km} \mathrm{sec}^{-1}$ to $\sim 5500 \mathrm{~km} \mathrm{sec}^{-1}$.

We model the first spectrum (23 days since $\mathrm{B}_{\max }$ ) of SN 2014dt. In Fig. 4, we present the observed spectrum with the output synthetic spectrum generated by the SYN++ code (Branch et al. 2007; Thomas et al. 2011). It was not possible to fit some of the broad emission features due to the use of the LTE approximation and the resonance scattering of this code. Fe II and Co II features match very well. Doublet at $4700 \AA$ due to Fe [III] lines, Co II and Fe II lines are fitted well. Co II and Fe II lines between $6700 \AA$ and $7700 \AA$ are also well produced. Although the NIR region is difficult to reproduce, the dip due to $\mathrm{Ca}$ is visible in the modelled 


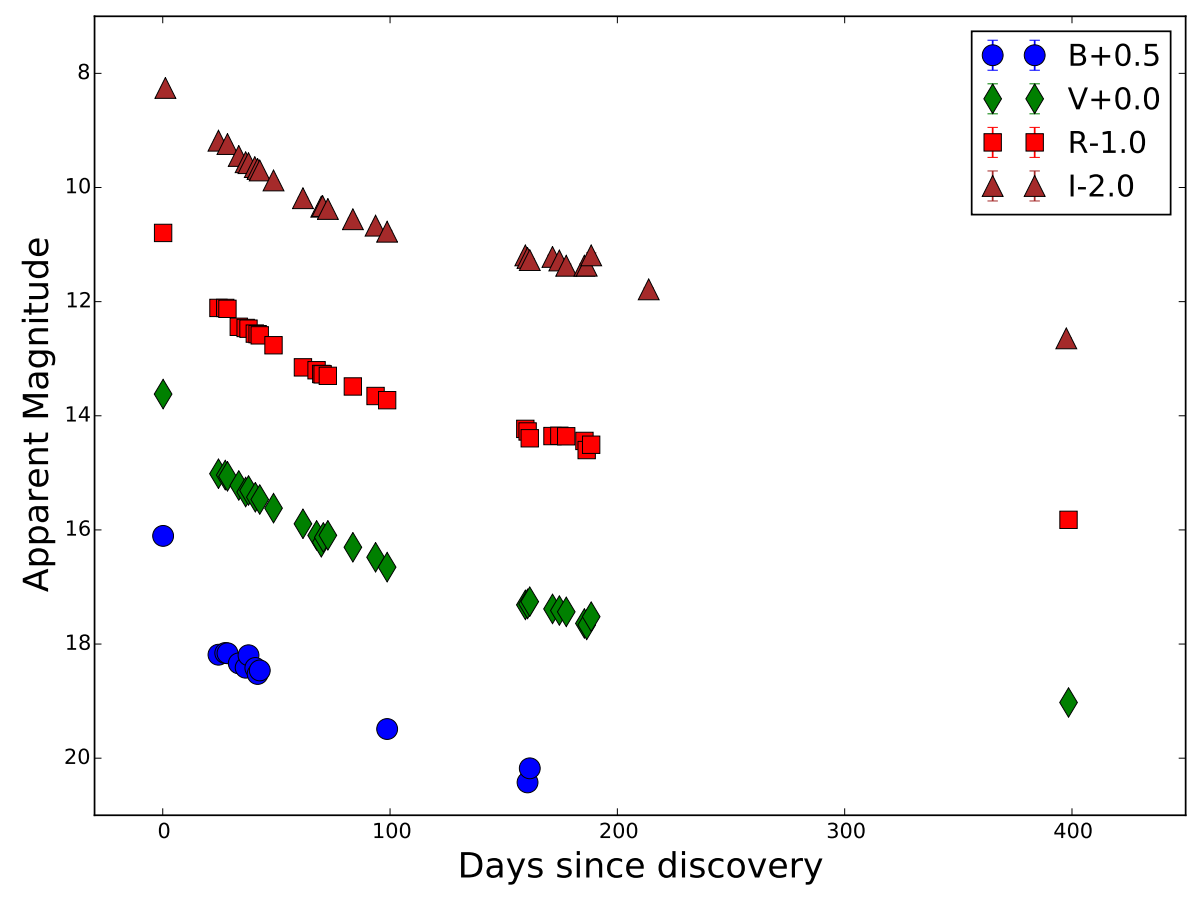

Figure 1: Broadband BVRI light curves of SN 2014dt. The light curves in different bands are shifted arbitrarily for clarity.

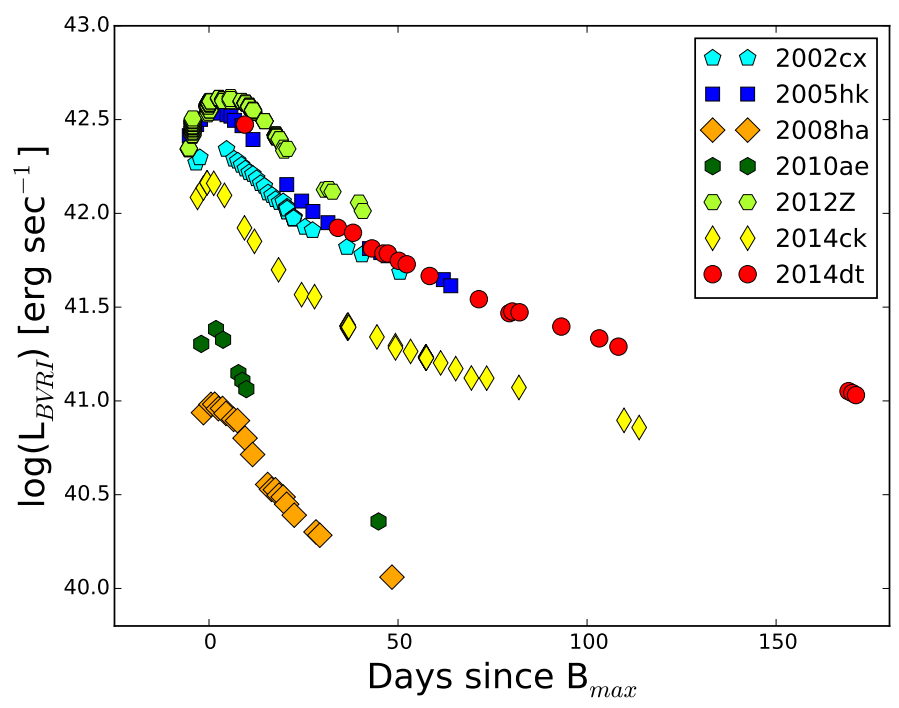

Figure 2: BVRI quasi bolometric light curve of SN 2014dt. A comparison with BVRI quasi bolometric light curves of other Type Iax SNe (SNe 2002cx (Li et al. 2003), 2005hk (Sahu et al. 2008), 2008ha (Foley et al. 2009), 2010ae (Stritzinger et al. 2014), 2012Z (Stritzinger et al. 2015; Yamanaka et al. 2015) and 2014ck (Tomasella et al. 2016)) shows that the luminosity of SN 2014dt is similar to SN 2005hk. 


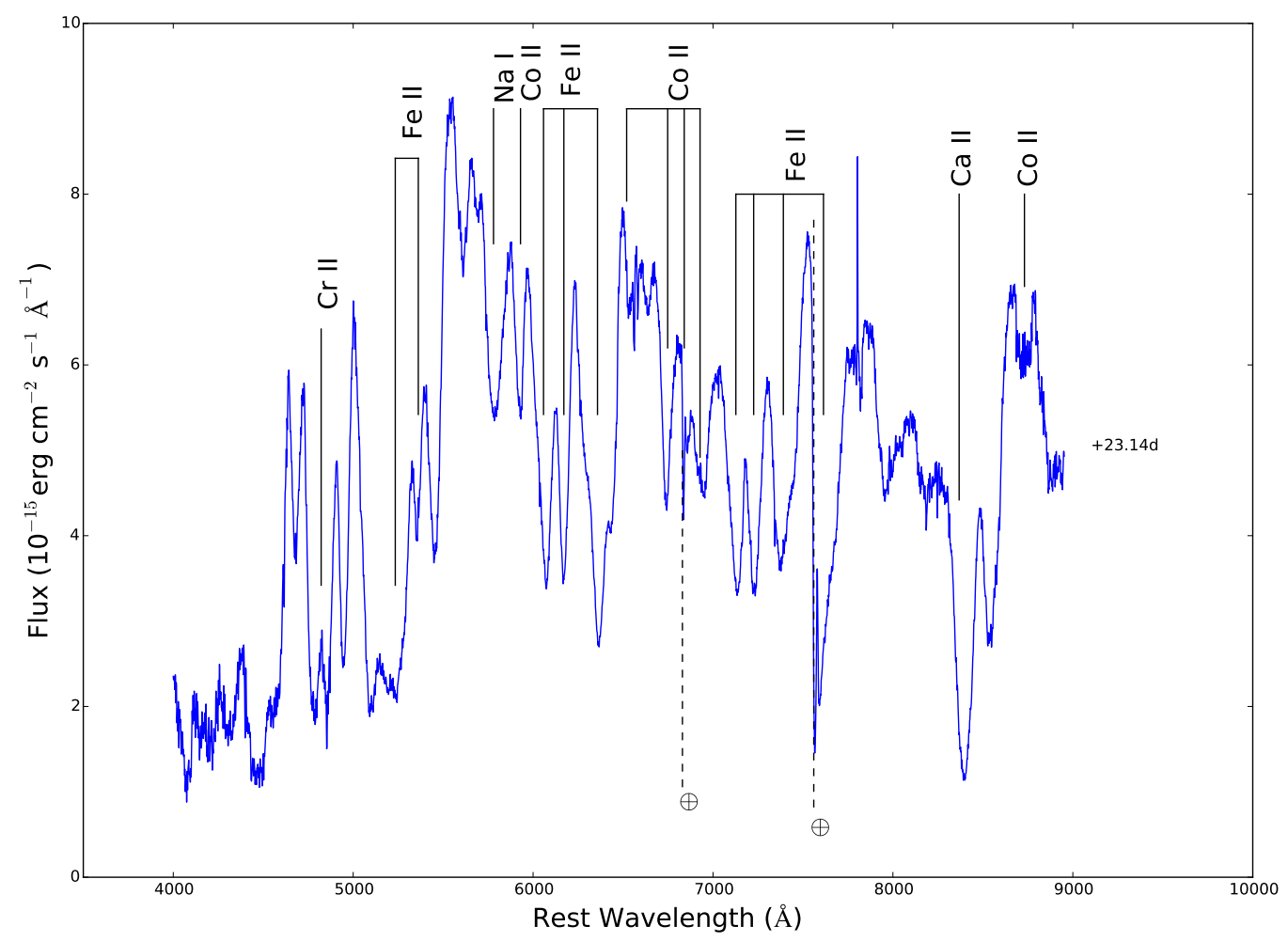

Figure 3: The earliest spectrum of SN 2014dt at 23 days is shown in the figure. Phase is calculated with respect to $\mathrm{B}_{\max }$. Telluric features are also shown in the figure.

spectra.

\section{Discussion}

Along with SNe 2008ge and 2012Z, SN 2014dt is the third member of the type Iax class with available preexplosion images. Light curve properties indicate towards a flattened structure. At very late epochs (298 to 326 days) a similar flattening is reported in the IR light curves of SN 2014dt by Fox et al. (2016). Up to 300 days the light curves of normal type Ia SNe are governed by the trapping of gamma rays after which the light curve transitions to the regime which is governed by the full trapping of positrons. Fox et al. (2016) argue that even a full positron trapping cannot fully explain the observed flattening seen in the IR light curves.

The IR excess seen in the late-time light curves implies the association with the pre-existence of a dust shell for the progenitor system which includes a binary system with a red giant, a red supergiant and an asymptotic giant branch star as a mass donor (Fox et al. 2016). Foley et al. (2016) claimed on the basis of their existing data that dust emission cannot be a source of excess IR flux. Instead, the plausible reason for strong IR flux seen about 315 days after maximum brightness is coming from a bound remnant with an extended optically thick super-Eddington wind. The bound remnant mechanism is consistent with the late-time data of type Iax SNe and could be the most likely progenitor system for SN 2014dt. Continued late-time observations of SN $2014 \mathrm{dt}$ will be necessary to conclude if the emission is dominated by the bound remnant.

SN 2008ha is the faintest member of this subclass for which a detection has been found at SN site four years after explosion. It is still an open question that the detection which was obtained at $\mathrm{SN}$ site is due to the remnant white dwarf or the companion star. With the photometry of the detection obtained four years after 


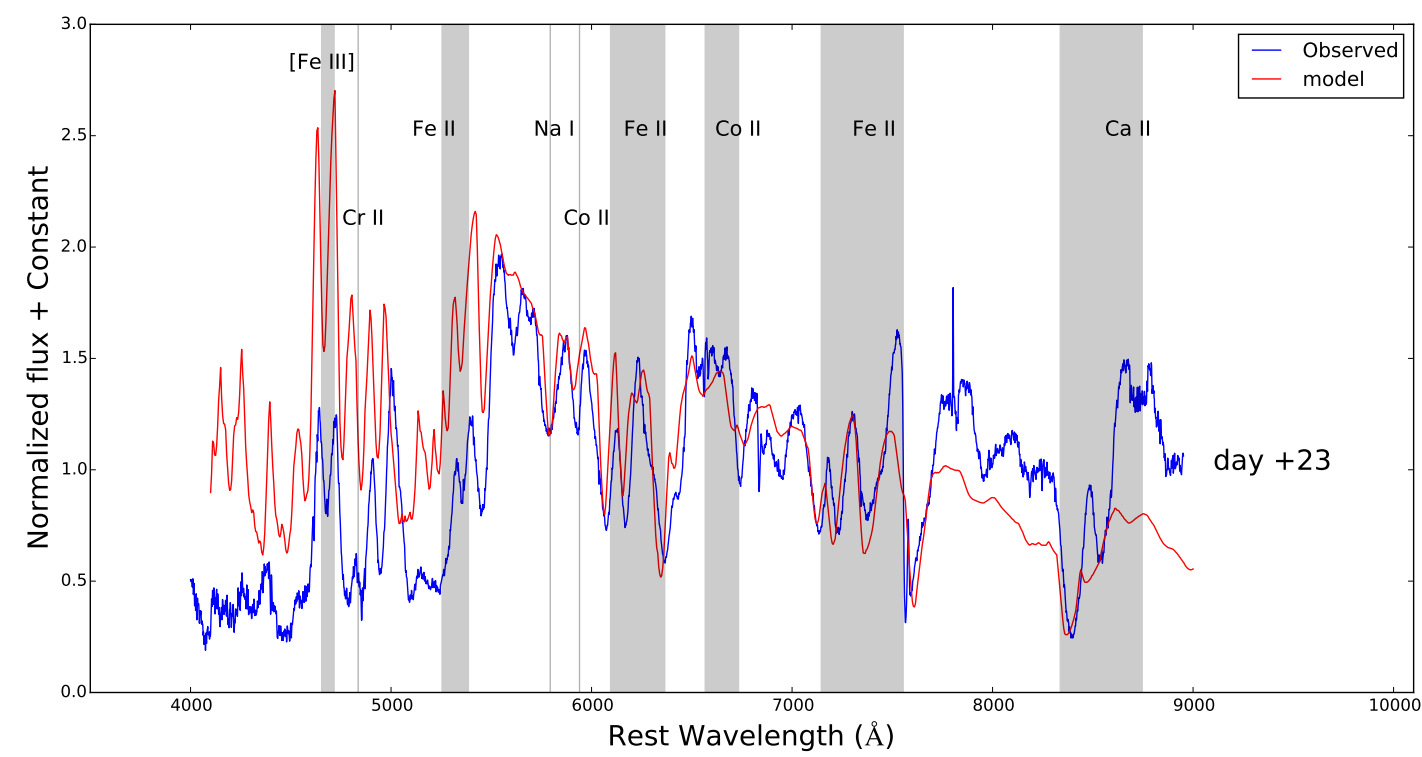

Figure 4: A comparison between the early phase observed spectrum of SN 2014dt and a synthetic spectrum generated by the SYN++ code. Prominent absorption features are marked with a shaded region.

the explosion it is proposed that it is an AGB star with an initial mass range $>3 \mathrm{M} \odot$. This mass range shows consistency with being the companion of a carbon oxygen white dwarf and not of a massive star. In such a case future observations are very necessary to confirm whether the detection is from the inflated remnant of a white dwarf or it is a companion star to the white dwarf only. If the late time emission is due to the remnant then it should vary on timescale of years and should have a peculiar spectrum. Moreover if it is only due to the companion star then its luminosity is unlikely to change with time.

If this is the case, deep imaging of SN 2014dt with larger telescopes in future will be very useful for detecting the possible bound remnant and putting constraints on the progenitor. In many cases, the chances of a late-time detection are rare mainly because they could be distant objects or relatively faint or the location of the SN is close to a bright host galaxy nucleus. The proximity of SN 2014dt and its location in the host galaxy are ideal for a late-time detection and makes this object as an excellent candidate for long term monitoring.

\section{Acknowledgements}

We thank the observing staff and observing assistants at $104 \mathrm{~cm} \mathrm{ST,} 130 \mathrm{~cm}$ DFOT and $201 \mathrm{~cm}$ HCT for their support during the observations of SN 2014dt. 


\section{References}

Arnett W. D. 1982, ApJ, 253, 785

Branch D., Parrent J., Troxel M. A. et al. 2007, in di Salvo T., Israel G. L., Piersant L., Burderi L., Matt G., Tornambe A., Menna M. T., eds, American Institute of Physics Conference Series Vol. 924, The Multicolored Landscape of Compact Objects and Their Explosive Origins. pp 342349, doi:10.1063/1.2774879

Foley R. J., Chornock R., Filippenko A. V. et al. 2009, AJ, 138, 376

Foley R. J., Challis P. J., Chornock R. et al. 2013, ApJ, 767, 57

Foley R. J., Van Dyk S. D., Jha S. W. et al. 2015, ApJ, 798, L37

Foley R. J., Jha S. W., Pan Y.-C., et al. 2016, MNRAS, 461, 433

Fox O. D., Johansson J., Kasliwal M. et al. 2016, ApJ, 816, L13

Li W., Filippenko A. V., Chornock R. et al. 2003, PASP, 115, 453

Li W., Leaman J., Chornock R. et al. 2011, MNRAS, 412, 1441

Liu Z.-W., Zhang J.-J., Ciabattari F. et al., 2015, MNRAS, 452, 838

Magee M. R., Kotak R., Sim S. A. et al. 2016, A\&A, 589, A89

Nugent P., Phillips M., Baron E., Branch D., Hauschildt P. 1995, ApJ, 455, L147

Phillips M. M., Li W., Frieman J. A. et al. 2007, PASP, 119, 360

Sahu D. K., Tanaka M., Anapuma G. C. et al. 2008, ApJ, 680, 580

Stetson P. B. 1987, PASP, 99, 191

Stritzinger M. D., Hsiao E., Valenti S. et al. 2014, A\&A, 561, A146

Stritzinger M. D., Valenti S., Hoeflich P. et al. 2015, A\&A, 573, A2

Szalai T., Vinkó J., Sárneczky K. et al. 2015, MNRAS, 453, 2103

Thomas R. C., Nugent P. E., Meza J. C. 2011, PASP, 123, 237

Tomasella L., Cappellaro E., Benetti S. et al. 2016, MNRAS, 459, 1018

van Dokkum P. G. 2001, PASP, 113, 1420

Yamanaka M., Maeda K., Kawabata K. S. et al. 2015, ApJ, 806, 191 